RESEÑA DE LIBRO

\title{
THE ELECTRIC FORCE OF A CURRENT: WEBER AND THE SURFACE CHARGES OF RESISTIVE CONDUCTORS CARRYING STEADY CURRENTS
}

by Andre Koch Torres Assis and Julio Akashi Hernandes 2007

(Available in pdf format at http://www.ifi.unicamp.br/ assis)

\author{
por H. Torres-Silva ${ }^{1}$
}

El Prof Andre K.T. Assis de la Universidad Estatal de Campinas en Brasil, en colaboración con el Prof. J. A. Hernandes, en este nuevo libro se ha centrado en refutar lo estipulado por R. Clausius, J. C. Maxwell, y otros, de que la supuesta falla en la detección de una fuerza entre una corriente de un hilo conductor estacionario y una carga casi estática invalida la ley fundamental de Weber.

En este libro se encuentra un buen material didáctico que es importante tener en cuenta en el desarrollo de algunas áreas del electromagnetismo y la ingeniería electromagnética, en sistemas donde la existencia de una fuerza entre un conductor y cargas estáticas tiene que ser considerada como un aspecto vital.

El objetivo de este libro es el análisis de la fuerza entre una carga puntual y un alambre resistivo llevando una corriente estacionaria, cuando están en reposo relativo y la carga es externa al circuito. Análogamente, se consideran y analizan el potencial y el campo eléctrico dentro y fuera de los conductores resistivos que llevan corrientes estacionarias. Los autores también debaten acerca de la distribución de cargas a lo largo de la superficie de los conductores, que generan este campo. Este es un tema importante para la comprensión de la circulación de corrientes a lo largo de los conductores. Lamentablemente, este tópico ha sido considerado de poca importancia por la mayoría de los autores que escriben sobre el electromagnetismo. El objetivo principal de este texto es presentar las soluciones a casos sencillos que se pueden resolver analíticamente, a fin de destacar las propiedades más importantes de este fenómeno.

Este texto está escrito para estudiantes de pregrado y posgrado en programas de: física, ingeniería eléctrica, matemáticas, historia y filosofía de la ciencia. Los capítulos 1, 2, 3, 5, 6, 7 y 8 son apropiados para estudiantes de pregrado. La intención de los autores es que este libro sea una contribución en la formación del pensamiento crítico en los estudiantes y de ayuda en la profundización de sus conocimientos en este ámbito fundamental de la ciencia.

Ellos comienzan mostrando que muchos importantes autores sostienen incorrectos puntos de vista con respecto a las corrientes estacionarias, no sólo en el pasado sino también en los últimos años. Luego discuten muchos experimentos que demuestran la existencia de una fuerza entre un conductor resistivo con una corriente constante y una carga externa en reposo relativo al conductor.

Este primer tema muestra que la electrodinámica clásica es un tópico no cerrado, en el que todavía hay mucho por descubrir. Otro objetivo es mostrar que la electrostática y las corrientes estacionarias están intrínsecamente conectadas. Los campos eléctricos dentro y fuera de los conductores resistivos que transportan corrientes estacionarias se deben a la distribución de las cargas mantenida por las baterías a lo largo de sus superficies. Esta presentación unifica los tratamientos de temas de electrostática y corrientes estacionarias, contrariamente a lo que se puede encontrar hoy en día en muchos textos básicos sobre estos temas.

Además del tratamiento electrostático, en capítulos posteriores se trata el tema de la inducción electrostática, imagen de cargas y temas relacionados. En particular, se muestra en detalle el cálculo de la fuerza entre un largo conductor cilíndrico y una carga puntual externa en reposo en relación al conductor.

1 Instituto de Alta Investigación. Universidad de Tarapacá. Arica, Chile. E-mail: htorres@uta.cl 
Los autores posteriormente tratan el tema principal del libro, considerando la fuerza entre un cable resistivo con una corriente constante y una carga en reposo en relación con el alambre y que está fuera del alambre. En particular, analizan la componente de esta fuerza que es proporcional a la tensión de la batería conectada al alambre (se discute la fuerza electromotriz de una batería, junto con su distinción del concepto de diferencia de potencial, en el capítulo 5 de este libro).

Se hace un análisis considerando primero conductores rectos de sección transversal arbitraria, en general, y describen un teorema general de cargas en su superficie. En seguida se trata el caso de un largo conductor recto de sección circular. Luego, se presenta el tratamiento de un cable coaxial y de una línea de transmisión. Posteriormente se ocupan de planos conductores y una cinta recta de anchura finita.

En la tercera parte del texto se consideran los casos en que la corriente cerrada sigue trayectorias curvadas a través de conductores resistivos. Inicialmente, se estudia de una cáscara cilíndrica con corriente azimutal. A continuación, se considera la corriente fluyendo en la dirección azimutal a lo largo de una carcasa esférica resistiva. Y, por último, se trata el caso de un conductor toroidal con corriente azimutal estacionaria. Aunque es mucho más complicado que los casos anteriores, esta última situación es extremadamente importante, ya que puede ser modelo de un circuito delimitado en un volumen finito del espacio cerrado llevando una corriente estacionaria cerrada, como un anillo resistivo. Esta tercera parte es apropiada para estudiantes de postgrado y puede ser utilizada también como texto complementario en cursos sobre electromagnetismo, circuitos eléctricos y métodos matemáticos de la física.

La intención de los autores en incluir soluciones analíticas básicas de todos estos casos en un único trabajo es hacer posible utilizar este material en cursos de pregrado o postgrado antes mencionados. Aunque los tratamientos y los procedimientos matemáticos son más o menos parecidos en todos los casos, se presentan en detalle para conductores de diferentes formas, por lo que los capítulos se pueden estudiar independientemente el uno del otro. Pueden entonces ser fácilmente incorporados a los libros o textos estándares que tratan el electromagnetismo y los métodos matemáticos para científicos.

A modo de ejemplo, en el tradicional plan de estudios de Electricidad y Magnetismo, electrostática y los circuitos son tratados como temas casi completamente separados. Fenómenos electrostáticos se analizan en términos de carga y campo, pero los circuitos se analizan en términos de corrientes y potenciales, y la conexión entre estos dos conjuntos de conceptos no se hace evidente. Esta disociación debilita la afirmación de que la física puede analizar una amplia gama de fenómenos a partir de un pequeño número de poderosos principios fundamentales. Este libro es una ayuda para comprender macro-micro conexiones y la integración de la electrostática y circuitos.

Este libro permite participar a los estudiantes en el análisis de circuitos desde un punto de vista microscópico, en términos de la relación entre la velocidad de deriva, la movilidad de las cargas, y la intensidad de campo eléctrico. Se puede mostrar que el campo interior de los circuitos se debe no sólo a las cargas dentro y fuera de la batería, sino también a las relativas a las superficies de los elementos de circuito. Los estudiantes pueden aprender a analizar circuitos DC y AC directamente en términos de interacción de Coulomb y la naturaleza atómica de la materia. Este análisis en términos de carga y campo hace una fuerte conexión con electrostática, unificando los dos temas, y proporciona un fuerte sentido de mecanismo físico para el comportamiento de circuitos simples.

Al final de este trabajo los autores presentan preguntas abiertas y describen perspectivas futuras. Los últimos capítulos en el libro están dedicados a los cálculos teóricos relacionados con la fuerza de Weber, que incluye un original tratamiento de la cáscara esférica resistiva. Un apéndice, "Wilhelm Weber y cargas de superficie", contiene un penetrante estudio de un importante trabajo de Weber en la Serie de medidas electrodinámicas, dedicada a la medición de la resistencia.

Un segundo apéndice, sobre la derivación de Gustav Kirchoff de la ecuación telegráfica, en la que se demuestra que la propagación de la corriente en un cable estaría limitada por la velocidad de la luz, fija las cosas que tanto Weber como Kirchoff había precedido por varios años a Maxwell en este descubrimiento.

Estos últimos capítulos pueden ser apropiados para la Investigación y el Desarrollo. Un caso es el efecto Aharonov-Bohm que normalmente se cree que es una indicación de la importancia fundamental de los potenciales electromagnéticos en la 
naturaleza. Aunque varias explicaciones no convencionales de este efecto se sugieren en la literatura, por lo que se sabe no hay intentos de interpretar este efecto en términos de interacciones directas entre las partículas. Se estima que con este material del libro, se puede llenar esta laguna para sugerir una simple descripción del efecto Aharonov Bohm dentro de la teoría de acción a distancia de Weber. Esta explicación no implicaría los conceptos de potenciales electromagnéticos y topologías espaciales no triviales. 